\title{
PREVALENCE OF CARDIAC AUTONOMIC NEUROPATHY IN TYPE 2 DIABETES MELLITUS
}

Samuel Dinesh Alfred ${ }^{1}$, Sukanya Nadinarajan², Sindhiya Jayachandran³, Pravin Selvam Selvaraj4, Santhi Chinnaiyan 5 , Kishore Raja Subramanian6, Vignesh Nariyamattu Chandrakumar7, Shivamalarvizhi ${ }^{8}$

\author{
${ }^{1}$ Associate Professor, Department of Internal Medicine, Stanley Medical College. \\ ${ }^{2}$ Assistant Professor, Department of Internal Medicine, Stanley Medical College. \\ 3 Postgraduate, Department of Internal Medicine, Stanley Medical College. \\ ${ }^{4}$ Postgraduate, Department of Internal Medicine, Stanley Medical College. \\ 5 Postgraduate, Department of Internal Medicine, Stanley Medical College. \\ 6 Intern, Department of Internal Medicine, Stanley Medical College. \\ 7 Intern, Department of Internal Medicine, Stanley Medical College. \\ ${ }^{8}$ Postgraduate, Department of Internal Medicine, Stanley Medical College.
}

\section{ABSTRACT}

\section{BACKGROUND}

Diabetic neuropathy, a set of clinical syndrome sometime silent and undetected, may be single or combined with signs which are nonspecific, insidious and slow and often diagnosed by exclusion. Neurologic complications occur equally in all types of diab etes type I, type II and all other types of diabetes.

\section{MATERIALS AND METHODS}

This study aimed at assessing the prevalence of Cardiac Dysautonomia in type 2 diabetic individuals using conventional testing methods and by using Ansiscope.

\section{RESULTS}

'At diagnosis' $68.8 \%$ were CAN positive by ansiscope and $81.2 \%$ by conventional method. In individuals with a duration of 1 - 5 years, $82 \%$ and $88 \%$ tested positive for autonomic dysfunction by ansiscope and conventional method respectively.

\section{CONCLUSION}

Diabetics for more than 5 years were tested $100 \%$ positive for autonomic dysfunction by either of the methods.

\section{KEYWORDS}

Diabetes, Ansiscope, Cardiac Autonomic Neuropathy.

HOW TO CITE THIS ARTICLE: Alfred SD, Nadinarajan S, Jayachandran S, et al. Prevalence of cardiac autonomic neuropathy in type 2 diabetes mellitus. J. Evolution Med. Dent. Sci. 2016;5(92):6854-6858, DOI: 10.14260/Jemds/2016/1550

\section{BACKGROUND}

\section{Diabetes: A Challenge of $21^{\text {st }}$ Century}

Change in the lifestyle of people along with globalisation in the past century has increased the incidence of diabetes. Findings of a report by Ramachandran et al has proved(1) urbanisation of India causing a high prevalence of diabetes. It has increased from 13.9 in 2002 to 18.2 in 2006 in urban areas. The rural area also showed an increase in prevalence from 6.4 in 2002 to 9.2 in 2006. According to International Diabetic Federation Diabetes Atlas,(2) India is next only to china hosting the high number of people with diabetes. The number of people with diabetes is expected to increase from 65.1 million in 2013 to 109 million in 2035 in India. Indians develop diabetic complications at an early age. This results in increase in mortality and morbidity among Indians. Despite a high prevalence of diabetes in South-East Asian countries, only 5\% of the global health care cost goes towards diabetes care.

Financial or Other, Competing Interest: None.

Submission 07-10-2016, Peer Review 06-11-2016,

Acceptance 12-11-2016, Published 17-11-2016.

Corresponding Author:

Dr. Samuel Dinesh Alfred,

No. 404, Shruthi Block, Chitra Avenue,

Choolaimedu High Road,

Chennai-94.

E-mail: meyappansindhi@gmail.com

DOI: $10.14260 /$ jemds $/ 2016 / 1550$

\section{(c) $($ ) $\odot$}

Prevention of complications associated with diabetes is achieved by primary prevention by modifying risk factors, such as insulin resistance and obesity.(3)(4)

Type 2 diabetes is a disorder characterised by insulin resistance, relative decrease in insulin secretion and hyperglycaemia. Environmental and genetic factors play a role in the development of diabetes.

Diabetic neuropathy, a set of clinical syndrome sometime silent and undetected, may be single or combined with signs which are non-specific, insidious and slow and often diagnosed by exclusion. Neurologic complications occur equally in all types of diabetes - type I, type II and all other types of diabetes.

Diabetic neuropathy is the most common cause of hospitalisation than other known cause of complications. So early diagnosis of cardiac autonomic neuropathy by using simple non-invasive investigation - heart rate variability using ECG helps in the identification of individuals at risk. This study aimed at assessing the prevalence of Cardiac Dysautonomia in type 2 diabetic individuals using conventional testing methods and by using mathematical instrument - Ansiscope.(5)

\section{MATERIALS AND METHODS}

Patients with Type 2 diabetes in both OP and IP basis from Feb. 2013 to Dec. 2014 were included in the study. Patients were subjected to symptom analysis, clinical examination and laboratory investigations. 
Eligible cohorts were subjected to standard autonomic testing and testing with Ansiscope.

Standard Autonomic Testing includes,

Assessing Heart Rate Variability with

1. Deep breathing.

2. Valsalva.

3. Supine-to-standing position.

Assessing Blood Pressure Variability with

1. Supine-to-standing.

\section{Procedure}

Resting ECG was taken for all the patients. Individuals in the study group were subjected to ECG recordings. Preferred lead is lead II.

Subjects were made to lie supine comfortably. Then they were asked to take deep breathe evenly at the rate of 6 breaths per minute, i.e. 5 seconds for inspiration and 5 seconds for expiration. A continuous ECG was recorded for one minute. The maximum and minimum $\mathrm{R}-\mathrm{R}$ interval during the respiratory cycle was calculated and converted to beats per minute. The difference between the two and heart rate variation of less than 10 beats per minute was taken as abnormal. Then the patient was allowed to lie quietly for another 5 minutes. The patient was made to exhale forcibly into the mouth piece of manometer sustaining a pressure of 40 mmHg for about 15 seconds and the ECG was recorded continuously. The patient was made to stop the manoeuvre and the ECG was further recorded post manoeuvre. The ratio of shortest R-R interval during the manoeuvre and the longest $\mathrm{R}-\mathrm{R}$ post valsalva was calculated. A ratio less than 1.10 was considered abnormal. Again the patient was made to lie supine quietly. After about 5 minutes with continuous monitoring, the patient was made to stand. The R-R internal at $15^{\text {th }}$ beat and $30^{\text {th }}$ beat was calculated. The $30: 15$ ratio of less than 1.00 was considered abnormal.

For the assessment of the sympathetic function, patient was made to lie down and his BP recorded. Then he was made to stand up and again BP measurement was made 2 minutes after standing. A fall of systolic Blood Pressure more than 30 mmHg was considered abnormal. Based on the above standard testing, patients with 2 or more abnormal tests were classified as definite, one of the three heart rate variability test abnormal were classified as early. When individuals with parasympathetic dysfunction along with significant BP fall were classified as severe CAN as per Ewings et al.

\section{Observations and Data Analysis} Sex Distribution

\begin{tabular}{|c|c|c|}
\hline Sex & Frequency & \% of Study Group \\
\hline Male & 30 & 35.7 \\
\hline Female & 54 & 64.3 \\
\hline Total & $\mathbf{8 4}$ & $\mathbf{1 0 0}$ \\
\hline
\end{tabular}

\section{CAN Positivity}

\begin{tabular}{|c|c|c|c|c|}
\hline \multirow{2}{*}{ CAN } & \multicolumn{2}{|c|}{ Ansiscope } & \multicolumn{2}{c|}{ Conventional } \\
\cline { 2 - 5 } & Frequency & $\mathbf{\%}$ & Frequency & $\mathbf{\%}$ \\
\hline Yes & 70 & 83.3 & 75 & 89.3 \\
\hline No & 14 & 16.7 & 9 & 10.7 \\
\hline Total & $\mathbf{8 4}$ & $\mathbf{1 0 0}$ & $\mathbf{8 4}$ & $\mathbf{1 0 0}$ \\
\hline
\end{tabular}

\section{Severity of CAN}

\begin{tabular}{|c|c|c|c|c|}
\hline \multirow{2}{*}{} & \multicolumn{2}{|c|}{ Ansiscope } & \multicolumn{2}{c|}{ Conventional } \\
\cline { 2 - 5 } & Frequency & $\mathbf{\%}$ & Frequency & \% \\
\hline Early & 9 & 12.8 & 19 & 25.0 \\
\hline Late & 47 & 67.1 & 53 & 69.7 \\
\hline Advanced & 14 & 20 & 4 & 5.26 \\
\hline Total & $\mathbf{7 0}$ & $\mathbf{1 0 0}$ & $\mathbf{7 6}$ & $\mathbf{1 0 0}$ \\
\hline
\end{tabular}

Valsalva Ratio

\begin{tabular}{|c|c|c|}
\hline & Frequency & Percentage \\
\hline Normal & 16 & 19 \\
\hline Borderline & 45 & 53.6 \\
\hline Abnormal & 23 & 27.4 \\
\hline Total & $\mathbf{8 4}$ & $\mathbf{1 0 0}$ \\
\hline
\end{tabular}

E:I Ratio

\begin{tabular}{|c|c|c|}
\hline & Frequency & Percentage \\
\hline Normal & 9 & 10.7 \\
\hline Borderline & 15 & 17.9 \\
\hline Abnormal & 60 & 71.4 \\
\hline Total & $\mathbf{8 4}$ & $\mathbf{1 0 0}$ \\
\hline
\end{tabular}

\section{0:15 Ratio}

\begin{tabular}{|c|c|c|}
\hline & Frequency & Percentage \\
\hline Normal & 11 & 13.1 \\
\hline Borderline & 20 & 23.8 \\
\hline Abnormal & 53 & 63.1 \\
\hline Total & $\mathbf{8 4}$ & $\mathbf{1 0 0}$ \\
\hline
\end{tabular}

\section{Orthostatic Hypotension}

\begin{tabular}{|c|c|c|}
\hline & Frequency & Percentage \\
\hline Normal & 59 & 70.2 \\
\hline Borderline & 21 & 25.0 \\
\hline Abnormal & 4 & 4.8 \\
\hline Total & $\mathbf{8 4}$ & $\mathbf{1 0 0}$ \\
\hline
\end{tabular}

\section{Sensitivity and Specificity of the HRV Test}

\begin{tabular}{|c|c|c|}
\hline Test & Sensitivity (\%) & Specificity (\%) \\
\hline Valsalva Ratio & 86.7 & 66.7 \\
\hline E:I Ratio & 92 & 33.3 \\
\hline 30:15 Ratio & 92 & 55.6 \\
\hline
\end{tabular}


Association of Various Parameters with CAN

\begin{tabular}{|c|c|c|c|c|c|}
\hline \multirow{2}{*}{ Parameter } & \multirow{2}{*}{$\%$ of Subjects } & \multicolumn{2}{|c|}{ Prevalence of CAN } & \multicolumn{2}{|c|}{ P Value } \\
\hline & & Conventional & Ansiscope & Conventional & Ansiscope \\
\hline Smokers & $15.5 \%$ & $100 \%$ & $92.3 \%$ & 0.384 & 0.58 \\
\hline Hypertension & $42.9 \%$ & $91.9 \%$ & $86.1 \%$ & 0.799 & 0.761 \\
\hline Peripheral Neuropathy & $32.1 \%$ & $96.3 \%$ & $92.6 \%$ & 0.293 & 0.210 \\
\hline \multicolumn{6}{|l|}{ Duration of Diabetes } \\
\hline a. At Diagnosis & $19 \%$ & $81.2 \%$ & $68.8 \%$ & \multirow{3}{*}{0.344} & \multirow{3}{*}{0.106} \\
\hline b. 1 - 5 years & $59.5 \%$ & $88 \%$ & $82 \%$ & & \\
\hline c. $>5$ years & $21.5 \%$ & $100 \%$ & $100 \%$ & & \\
\hline Unaware & $75 \%$ & $93.7 \%$ & $85.7 \%$ & 0.039 & 0.049 \\
\hline Poor Sugar Control & $33.33 \%$ & $100 \%$ & $96.4 \%$ & 0.026 & 0.029 \\
\hline
\end{tabular}

\section{RESULTS}

1. Among the total 84 individuals studied, $64.3 \%$ were females and $35.7 \%$ were males.

2. A total of $15.5 \%$ (13 persons) were smokers and among them $92.3 \%$ (12 persons) and about 100\% (13 people) were found to have cardiac autonomic neuropathy by ansiscope and conventional methods respectively.

3. 27 subjects, i.e. $32 \%$ were found to have peripheral neuropathy by clinical testing and among them 92.6\% (25 persons) tested positive for autonomic neuropathy by ansiscope and $96.3 \%$ (26 persons) by conventional method.

4. 36 subjects (42.9\%) of the study population were hypertensive and of them 31 people (86.1\%) and 33 people $(91.7 \%)$ tested positive for autonomic dysfunction by ansiscope and conventional method respectively.

5. Of the 84 study subjects, 63 (75\%) people were not aware of the complications related to diabetes. In them 54 subjects (85.7\%) and 59 subjects (93.7\%) tested positive for cardiac autonomic neuropathy by ansiscope and conventional methods respectively. This association is found to be statistically significant.

6. A total of 16 subjects (19\%) were examined at diagnosis. Among them 11 individuals $68.8 \%$ were CAN positive by ansiscope and $81.2 \%$ (13 subjects) by conventional method.

7. Of the 84 individuals 50 individuals were diabetic for a duration 1 - 5 years. Among them, 82\% (41 individuals) and $88 \%$ (44 subjects) tested positive for autonomic dysfunction by ansiscope and conventional method respectively.

8. Of the 18 subjects who were diabetic for more than 5 years were tested $100 \%$ positive for autonomic dysfunction by both the methods.

9. 28 individuals had poor glycaemic control. All individuals with poor glycaemic control tested positive for cardiac autonomic dysfunction by conventional method and $96.4 \%$ (27 individuals) tested positive by ansiscope. This association is statistically significant.

\section{Pathogenesis}

Free radicals - a free radical is a species which has one or more unpaired free electron in its orbit.

\section{Mechanism of Generation of Oxygen Free Radicals}

Electron transfer reactions like hydroxyl radicals, superoxide anion radical, lipid alkoxyl and peroxyl radical and hydrogen peroxide generate free radicals.
Energy transfer reactions like triplet carbonyl compounds and singlet oxygen are also involved in generation of free radicals.

\section{Hyperglycaemia-Induced Mitochondrial Superoxide Production}

During electron transfer in respiratory chain, a proton gradient is created by extrusion of protons into intermembrane space of mitochondria.

This gradient stimulates ATP synthase. In diabetes with high intracellular glucose concentration, more glucose is oxidised via citric acid cycle and electron donors like NADH, FADH2 gets used up. After a critical threshold, the electron transfer inside complex 3 gets blocked. This process produces electron to get backed-up to coenzyme- $Q$, which donates electron to oxygen producing superoxide anion.

Hyperglycaemia activates other pathways like redox changes, NADPH oxidases and uncoupled eNOS gets amplified and produce superoxide.

Hyperglycaemia-Induced Mitochondrial Superoxide Production - Activates other Pathways by Inhibiting GAPDH(5)(6)

Intracellular hyperglycaemia reduces the glycolytic enzyme GAPDH. This causes other glycolytic intermediates to increase.

1. High levels of glyceraldehyde 3 phosphate - glycolytic metabolite activates following two pathways.

a. AGE pathway - glyceraldehyde 3 phosphate is the source for AGE precursor methylglyoxal.

b. Classic protein kinase C (PKC) pathway: glyceraldehyde 3 phosphate is the source for diacylglycerol, which is the activator of PKC pathway.

2. Levels of fructose 6 phosphate increase, which is a glycolytic metabolite activates hexose amine pathway to form UDP-GlcNAc.

3. GADPH inhibition also increases the intracellular glucose level, which enters polyol pathway.

a. Sorbitol is formed from glucose by the enzyme aldolase reductase consuming NADPH. Increased sorbitol is neurotoxic causing Schwann cell damage by increasing cell osmolarity.

b. Depletion of NADPH in the above process decreases intracellular myoinositol, which interfere with cellular metabolism. 


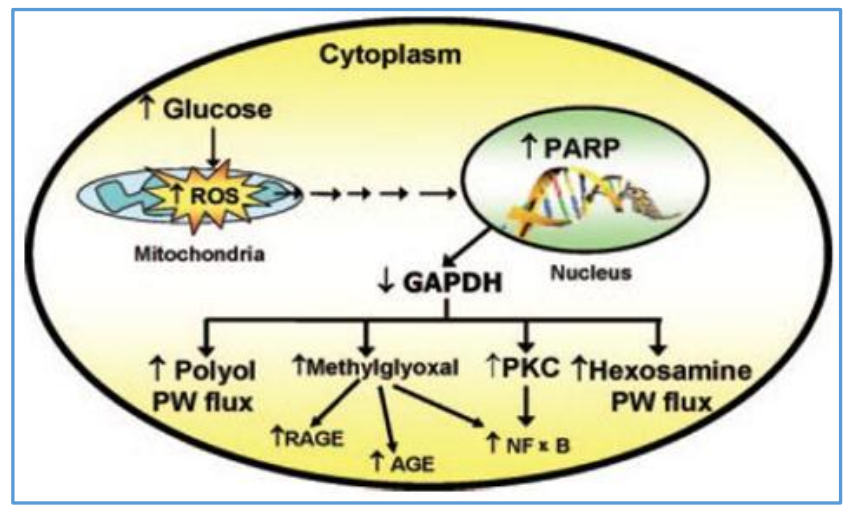

Mechanism of Hyperglycaemia-Induced Cellular Damage

Cellular Injury due to Increased Reactive Oxygen Species

1. Oxygen free radicals attack the iron-sulphur moiety of enzymes and proteins and inhibit them. The proteins more susceptible for inhibition are complexes I - III of electron transfer chain, biotin synthase and aconitase of citric acid cycle.

2. Lipids in membranes of mitochondria, plasma and endoplasmic reticulum undergoes peroxidation. The end products of this process - lipid peroxides are toxic to the cell.

3. Proteins and nucleic acid in the cells undergo peroxidation and nitrosylation, which are toxic to the cell.

4. Oxidative modification of various transcription factor causes reduced expression of anti-apoptic proteins like Bcl-2 and increase in pro-apoptic proteins.

5. Oxidative damage of DNA, especially in non-dividing cells like neurons affect axonal transport and signalling resulting in loss of function of neurons.

\section{NRF2 and Oxidative Stress}

NRF2 is a transcription factor that protects against oxidative stress. NRF2 expression is down regulated in diabetic nerves. DRG neurons are protected from free radical injury via NRF2 activation. Hence, hyperglycaemia-induced downregulation of NRF2 makes Schwann cells and DRG more susceptible to oxidative stress.

Role of Advanced Glycation End Products in the Pathogenesis of Diabetic Neuropathy

\section{AGEs}

AGE are heterogeneous compounds, which are formed by nonenzymatic glycation and oxidation of proteins and/or lipids with aldose sugars.

\section{Formation of AGE}

Aldehyde of glucose combines with amino acid side chain and forms covalent bond, which is labile. Early glycation produces Schiff basis, which are reversible. These undergo further glycation and Amadori rearrangement to produce irreversible AGE products.

\section{Examples}

Pentosidine, Glycated HbA1C, N-carboxymethyl lysine, Methylglyoxal.

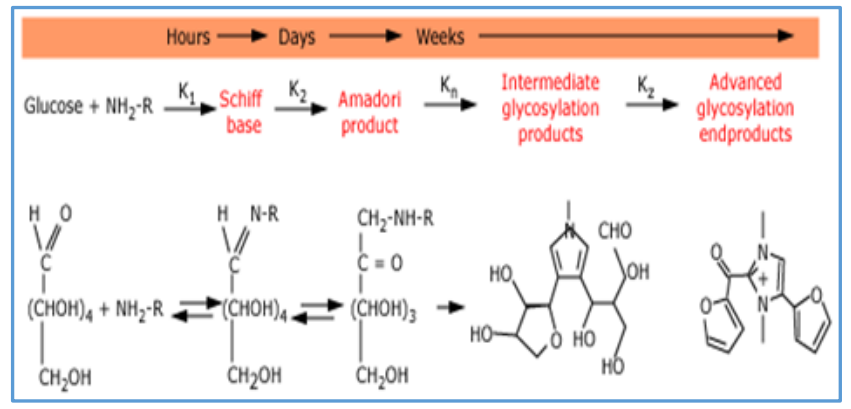

Formation of Advanced Glycation End Products

\section{Receptor for Age (RAGE)}

RAGE belongs to immunoglobulin receptor super family. They are expressed minimally in normal non-diabetic tissue and vessels. Chronic hyperglycaemia produces more AGE products, which by feedback mechanism upregulates RAGE expression. RAGE stimulation produces pro-inflammatory response.

\section{AGE on Function of Extracellular Tissue}

1. AGE produces cross-linking of elastin and type I collagen, thereby producing increased stiffness of vessels.

2. AGE interaction via RAGE decreases binding heparin sulfate, a proteoglycan in the vessel wall to the basement membrane producing a procoagulant state.

3. Glycation of LDL decreases nitrous oxide, thereby decreasing vasodilatation.

4. Glycated LDL decreases clearance and uptake of LDL producing a pro-atherogenic state promoting smooth muscle cell proliferation and atheroma formation in endoneurial vessels.

\section{AGE on Function of Intracellular Milieu}

1. Glycation of FGF affects vascular homeostasis.

2. AGE-induced stimulation of monocytes and endothelial cells causes increased expression of E-selectin, VEGF, VCAM-1, ICAM-1, other pro-inflammatory cytokines like IL-1, IL-6, TNF-A, RAGE.

\section{Role of AGE in Diabetic Neuropathy(7)(8)}

1. AGE/RAGE induce oxidative stress that increases glycosidation products like pentosidine.

2. Upregulation of nuclear product NF - kappa B and various pro-inflammatory genes alter neurological function.

3. Atherosclerotic endoneurial vessels produces ischaemic nerve damage.

4. Hyperglycaemia-induced AGE causes segmental demyelination of peripheral nerves.

5. AGE alters cytoskeletal proteins of axons like tubulin, actin, neurofilament and produces atrophy of axons and degeneration.

6. Glycation of laminin produces reduced axonal regeneration.

7. Endoneurial deficiency of nitrous oxide affects microvasculature.

\section{Polyol Pathway}

Peripheral nerves uptakes glucose in a non-insulin dependant manner. In hyperglycaemia, high glucose in the nerves enter polyol pathway. 


\section{Two Enzymes are involved in the above Pathway}

a. Aldose Reductase (AR) - in the presence of cofactor NADPH reduces glucose to sorbitol.

b. Sorbitol Dehydrogenase (SDH) - in the presence of cofactor NAD + forms fructose from sorbitol.

1. Sorbitol oxidation by SDH produces NADH from NAD+. $\mathrm{NADH}$ acts as a substrate for NADH oxidase to produce ROS.

2. Fructose formed is converted to 3 deoxyglucosone and fructose 3 phosphate. These are extremely potent nonenzymatic glycation agents. AGE acts via RAGE causing oxidative stress. Protein kinase $\mathrm{C}$ pathway.

Hyperglycaemia increases diacylglycerol, which activates PKC. Active PKC increases expression of TGF-B and other proinflammatory cytokines, which produces oxidative damage of the diabetic nerves.(8)

\section{Hexosamine Pathway}

Fructose 6-phosphate is converted to glucosamine 6phosphate by hexosamine.

\section{CONCLUSION}

The prevalence of cardiac autonomic neuropathy is extremely high among diabetics as shown in our study. Most diabetic patients have significant autonomic dysreflexia even at diagnosis of diabetes. Cardiac autonomic neuropathy is the single important factor, which predicts the 5-year mortality rate among diabetics. Poor blood sugar control is significantly associated with CAN and is the single best factor, which determines the rate of progression as cited in our study. Early screening, early diagnosis, proper education of patients and strict glycaemic control help in the arrest of progression of Cardiac Autonomic Neuropathy in Type 2 diabetic population.

\section{REFERENCES}

1. Ramachandran A, Mary S, Yamuna A, et al. High prevalence of diabetes and cardiovascular risk factors associated with urbanization in India. Diabetes Care 2008;31(5):893-8.

2. Prior IA, Davidson F. The epidemiology of diabetes in Polynesians and Europeans in Newzealand and Pacific. N Z med J 1966;65(406):375-83.

3. Ramachandran A, Snehalatha C. Diabetes prevention and control programme in developing countries. In: Narayan KMV, Williams D, Gregg EW, et al. eds. Diabetes Public Health. Oxford Univ Press 2011:603-21.

4. InterAct Consortium, Scott RA, Langenberg C, et al. The link between family history and risk of type 2 diabetes is not explained by anthropometric, lifestyle or genetic risk factors: the EPIC-InterAct study. Diabetologia 2013;56(1): 60-9.

5. Meigs JB, Cupples LA, Wilson PW. Parental transmission of type 2 diabetes: the Framingham Offspring Study. Diabetes 2000;49(12):2201-7.

6. Shai I, Jiang R, Manson JE, et al. Ethnicity, obesity and risk of type 2 diabetes in women: a 20-year follow-up study. Diabetes Care 2006;29(7):1585-90.

7. DeFronzo RA, Ferrannini E. Insulin resistance. A multifaceted syndrome responsible for NIDDM, obesity, hypertension, dyslipidemia and atherosclerotic cardiovascular disease. Diabetes Care 1991;14(3):173-94.

8. Reis JP, Loria CM, Sorlie PD, et al. Lifestyle factors and risk for new-onset diabetes: a population-based cohort study. Ann Intern Med 2011;155(5):292-9. 